\title{
Éducation et congrégations religieuses enseignantes en Italie pendant la Restauration
}

\section{Michel Ostenc}

\section{(2) OpenEdition \\ 1 Journals}

Édition électronique

URL : http://journals.openedition.org/assr/23400

DOI : $10.4000 /$ assr.23400

ISSN : $1777-5825$

Éditeur

Éditions de l'EHESS

\section{Édition imprimée}

Date de publication : 31 décembre 2011

Pagination : 69-84

ISBN : 9782713223273

ISSN : 0335-5985

\section{Référence électronique}

Michel Ostenc, «Éducation et congrégations religieuses enseignantes en Italie pendant la

Restauration ", Archives de sciences sociales des religions [En ligne], 156 | octobre-décembre 2011, mis en ligne le 30 janvier 2012, consulté le 30 avril 2019. URL : http://journals.openedition.org/assr/23400 ; DOl : 10.4000/assr.23400 


\section{Michel Ostenc}

\section{Éducation et congrégations religieuses enseignantes en Italie pendant la Restauration}

L'Italie a connu dans la première moitié du XIX siècle une véritable floraison de nouvelles congrégations religieuses qui s'ajoutèrent aux institutions traditionnelles durement éprouvées pendant la période révolutionnaire et napoléonienne. Plus de cent vingt de ces nouvelles fondations étaient féminines, sur les cent quarante qui furent créées entre 1800 et 1860, et les deux tiers se consacraient à l'éducation. Elles comptaient plus de dix mille religieuses sur les 42664 présentes en Italie en 1861. La grande majorité d'entre elles naquirent en Italie du nord et plus précisément en Lombardie-Vénétie autrichienne. L'étude du contexte politique et religieux de leur implantation, de la spiritualité de leurs fondateurs et de leur apostolat éducatif a fait l'objet de multiples travaux en Italie au cours des dix dernières années.

Dans le royaume de Piémont-Sardaigne, l'enseignement mutuel s'était développé à partir de 1818 et on lui devait quelques-unes des principales innovations pédagogiques de l'époque ; mais après les mouvements révolutionnaires de 1821 , le souverain Charles-Félix confia le système scolaire de ses États au jésuite Taparelli d'Azeglio qui lui imprima une forte connotation éthico-religieuse. Les Regie Patenti de 1822 autorisèrent les curés à enseigner dans leur paroisse, alors que les maîtres laïques devaient solliciter une autorisation de l'État, et elles ouvrirent largement les portes de l'enseignement aux congrégations religieuses. Les Oratoriens ouvraient des collèges à Biella et à Ivrée, les pères somasques à Casale Monferrato et les Barnabites à Verceil. Les Jésuites dispensaient un enseignement universitaire en Lettres, Droit ou Médecine à Chambéry, Nice et Novare. Certes, les écoles publiques restaient prépondérantes dans l'enseignement primaire avec un corps enseignant composé de prêtres et de laïcs; mais les Sœurs de Saint Joseph du Puy avaient créé des fondations autonomes à Turin, Cuneo et Novare, et les Frères des Écoles chrétiennes ne tardèrent pas à jouir d'une grande notoriété. Les conservateurs préféraient les établissements scolaires religieux et ils reprochaient à l'enseignement mutuel d'origines lancastériennes une logique utilitaire d'essence protestante incompatible avec la conception éthique et sociale du catholicisme. Les écoles des frères «Ignorantelli » enseignaient en langue vulgaire dans des classes de soixante élèves organisées en trois niveaux d'études 
pour la lecture, l'écriture et le calcul. Le maître s'occupait d'un seul écolier à la fois pendant que les autres s'adonnaient à des exercices répétitifs destinés à consolider les acquis. L'orientation conservatrice de la politique scolaire piémontaise fut progressivement abandonnée sous le règne de Charles-Albert, l'ascension de la bourgeoisie contribuant à diffuser des idées d'alphabétisation du peuple. Les initiatives en faveur des asiles pour la petite enfance s'inspiraient du prêtre libéral Ferrante Aporti qui avait ouvert le premier établissement de ce type en Italie dès 1828. Aporti restait silencieux sur ses relations avec les milieux qui soutenaient le mouvement des écoles enfantines en France et en Angleterre pour ne pas s'exposer aux critiques de l'Église ; mais l'aristocratie libérale de Turin n'était pas tenue à de telles précautions. Elle connaissait les salles d'asile de JeanMarie Denis Cochin, L'Éducation progressive de madame Necker de Saussure et le manuel d'enseignement primaire d'Ambroise Rendu. Ces influences avaient même incité le marquis de Barolo à ouvrir une salle d'asile pour enfants indigents dans son palais. Les libéraux piémontais des années 1840 reprochaient désormais aux frères des Écoles chrétiennes la place excessive des pratiques religieuses dans leurs écoles et leur enseignement n'était plus un modèle que pour les conservateurs.

Les catholiques piémontais étaient imprégnés d'esprit salésien pour des raisons historiques et géographiques. Cette spiritualité, faite d'optimisme, d'équilibre et de discrétion, se mêlait à celle de Philippe de Néri, pleine de foi dans la nature humaine, de simplicité et de mansuétude. Si bien qu'à partir des années 1840, les séminaires piémontais abandonnèrent la rigueur théologique pour une spiritualité plus encline à la compréhension et au pardon. Le prêtre devenait l'éducateur de ses ouailles et interprétait le message évangélique comme un soutien à la dignité humaine. Les idées pédagogiques d'Antonio Rosmini restauraient une conception de la connaissance comme illumination de l'esprit qui avait été ruinée par l'empirisme de la pensée du XVIII ${ }^{e}$ siècle. La formation du cœur sollicitait à la fois la volonté et le sentiment tout en restant étroitement associée à l'intelligence, les deux notions s'influençant réciproquement. La morale couronnait l'éducation rosminienne dont l'unité était garantie par l'universalité du principe chrétien. Ainsi, l'être réel s'unissait à l'être idéal dans une synthèse qui se nouait dans l'être moral. La promotion de l'éducation devenait un acte de foi chrétienne et le maître jouait un rôle décisif dans ce processus. Il était religieux et charitable, la foi permettant un libre accueil de l'être réel qui était charité. Rosmini fonda l'Institut de charité en 1828 et sa spiritualité animera la plupart des nouvelles congrégations religieuses piémontaises : les sœurs de la Providence, ou Rosminiennes, les sœurs de la Charité d'Ivrée et les sœurs de Sainte Anne fondées par la marquise de Barolo à Turin en 1834. Le style pastoral de Don Bosco, fait de compréhension et d'amour de la jeunesse, s'inspira largement de cet héritage spirituel. L'éducation du peuple revêtait une dimension morale et religieuse, tout en se référant à des options humaines toujours plus larges. La spiritualité de Don Bosco n'avait rien d'original ; mais elle le conduisit à un mariage heureux entre ecclésiologie théorique et ecclésiologie vécue. 
Aux portes de la Vénétie, Vérone fut rattachée en 1815 à un empire d'Autriche dont elle devint le principal point de contact avec l'Italie. Fortifiée par Napoléon qui en fit une place d'armes, la cité conservait des activités artisanales et commerciales. La domination française avait transformé ses nombreux couvents en casernes et imposé à la population un service militaire étranger à la mentalité de la population, multipliant ainsi les cas d'insoumission. Cette situation troublée qui s'ajoutait à la misère sociale contribua à accroître le nombre d'enfants illégitimes et abandonnés dans une ville au fort taux de mortalité infantile. La suppression de nombreux ordres religieux eut de profondes répercussions sur les missions caritatives de l'Église et la multiplication des nouvelles congrégations devait y remédier. L'enseignement pour les filles était une nouveauté pour l'époque, l'instruction féminine étant dispensée jusque-là dans les couvents et réservée aux demoiselles de l'aristocratie. La congrégation de Madeleine de Canossa joua un rôle pionnier dans ce domaine et ses écoles de charité, qui dispensaient un enseignement primaire et une formation professionnelle, se répandirent en Italie du nord ; mais son orientation sociale et son refus de la clôture provoquèrent la naissance à Vérone de la congrégation des sœurs de la Sainte Famille de Leopoldine Naudet qui entendaient concilier l'action éducative auprès des filles de la bourgeoisie avec la prière et la contemplation. Les écoles des sœurs de la Charité de Teodora Campostrini s'inspiraient de l'enseignement mutuel, mais uniquement par leur nombre limité d'élèves et par le tutorat des plus âgées sur leurs jeunes condisciples. La congrégation des Filles de Jésus fondée en 1812 par le père Pietro Leonardi était très engagée dans les missions sociales de l'Église véronaise. L'assistance charitable était sa principale activité, mais l'instruction n'en occupait pas moins une place importante dans ses missions car elle était considérée comme un moyen de rachat social et moral des classes pauvres. La congrégation des Filles de Jésus multipliait les écoles de charité qui entendaient libérer les filles pauvres de leur misère morale et matérielle. Elle avait adopté des règles peu contraignantes qui permettaient aux religieuses de regagner leur couvent le soir seulement, après une journée passée à l'extérieur pour remplir leur tâche. Les institutrices de Sainte Dorothée Filles du Sacré-Cœur, fondées à Vicence en 1840 par le père Giovanni Antonio Farina, étaient même autorisées à loger dans la paroisse où elles enseignaient. Les congrégations « canossiennes » ne pouvaient accepter de tels accommodements et elles décidèrent d'organiser des "séminaires " pour former des tertiaires de leur ordre, habilitées à enseigner dans les zones rurales trop éloignées des couvents. L'éducation des sourds-muets figurait également dans leur apostolat. Le langage des signes fut progressivement abandonné dans leurs écoles, le passage au langage articulé étant associé par le père Antonio Provolo à la pratique du chant.

Le Royaume lombard-vénitien connut, après 1815 , un retour à la politique scolaire réformiste du XVIII ${ }^{\mathrm{e}}$ siècle. Le règlement de 1818 s'efforça de compléter la diffusion de l'enseignement primaire et les écoles de secours mutuel se multiplièrent à l'initiative de Federico Confalonieri et des intellectuels libéraux rassemblés autour de la revue $\mathrm{Il}$ Conciliatore; mais le mouvement fut stoppé par la 
répression qui suivit les insurrections de 1821 et il déclina ensuite. L'Église lombarde avait été durement frappée par la politique joséphiste de suppression des couvents qui s'était poursuivie à l'époque révolutionnaire et napoléonienne; mais les ordres religieux traditionnels reprirent progressivement leurs activités et le règlement de 1818 accrut le rôle du clergé dans l'enseignement avec un droit de direction et d'inspection. L'enseignement de la doctrine catholique devait favoriser le contrôle idéologique des masses populaires. Les Jésuites rencontrèrent des obstacles presque insurmontables à leur retour en Lombardie-Vénétie et en Toscane, où les expériences du réformisme joséphiste avaient laissé des traces profondes. Ces États préféraient s'adresser aux pères somasques ou barnabites dont les ordres religieux s'étaient montrés plus ouverts aux réformes du despotisme éclairé ; mais les Barnabites, qui jouissaient pourtant du soutien de la noblesse et de la bourgeoisie milanaises, éprouvèrent beaucoup de difficultés pour retrouver leur siège historique de Saint Barnabé. Cependant, les diocèses de Milan, Bergame et Brescia enregistraient la plus forte concentration de nouvelles congrégations féminines. La précarité de l'emploi salarié, alliée aux épidémies et aux mauvaises récoltes, avait provoqué l'exode vers les villes lombardes d'une population rurale paupérisée. Le travail dans les manufactures modifiait profondément la morale des classes populaires et une tâche d'assistance auprès de l'enfance pauvre et abandonnée devenait une urgente nécessité. Des associations catholiques permirent la création de la congrégation des Sœurs de la Réparation qui se consacra aux filles délinquantes. Les congrégations féminines influencées par les Jésuites se spécialisèrent par contre dans l'éducation des filles de la noblesse. Ce fut le cas des Sœurs de Sainte Marcelline fondées à Milan en 1838 par le directeur spirituel du séminaire de Milan, Luigi Biraghi, et par Marina Videmari. Leurs « Règles » de 1853 consentaient une réduction des pratiques religieuses afin de faciliter leur contact avec le monde et l'originalité de leurs collèges venait des liens étroits entretenus avec les familles. Les jeunes filles pouvaient rentrer chez elles un jour par semaine et y passer leurs vacances d'été. Le but recherché était de maintenir un contact des élèves avec la société afin de mieux les préparer à la vie qui les attendait. Le «Colleggio Apostolico del Sacro Cuore» de Bergame insuffla son idéal éducatif à la congrégation des sœurs de la Sainte Famille d'Elisabetta Cerioli, aux sœurs de la Charité de Bartolomea Capitanio et aux Filles du Sacré-Cour de Jésus de Teresa Eustachio Verzeri. Dans le diocèse de Brescia, l'association des Amicizie cristiane présidait à la fondation de la congrégation des Filles de Marie Immaculée du chanoine Ludovico Pavoni, à celle des Servantes de la Charité et des Fils de l'Immaculée Conception. Enfin, à Vicence en Vénétie, un proche de Rosmini Luca Passi fondait une congrégation de Sainte Dorothée.

Il est vrai que le taux d'analphabétisme en Lombardie et Vénétie était l'un des plus bas de la péninsule et la multiplication des congrégations religieuses enseignantes répondait à une demande d'instruction toujours plus forte dans les zones déjà alphabétisées. Leur adaptation aux besoins de la population incitait 
ces congrégations à mettre l'accent sur l'enseignement féminin, les filles étant moins scolarisées que les garçons. Le renouveau de l'assistance charitable à l'enfance pauvre et abandonnée expliquait l'intérêt porté à l'éducation des sourds-muets. Les établissements les plus nombreux se situaient en LombardieVénétie où les nouvelles congrégations se montraient plus sensibles que les précédentes à la dimension sociale de la charité. Elles formaient leurs propres religieuses enseignantes qui choisissaient le type de formation le mieux adapté à leurs élèves. Ce fut le cas des filles de la Charité qui accueillirent les premières sourdes-muettes dans leurs écoles, les " canossiennes " s’intéressant à cet enseignement dès les années 1830. À Milan, l'abbé Giulio Tarra s'inquiétait d'un usage excessif du langage des signes et il inventa une méthode linguistique qui multipliait les exercices destinés à améliorer la prononciation, la parole devenant le moyen essentiel de la communication.

Les nouvelles congrégations s'intéressaient aussi à l'éducation de la jeunesse bourgeoise. Les Ursulines avaient toujours rempli ce rôle et Maria Maddalena Barioli reconstitua une congrégation dans cette tradition en 1841. L'originalité des Ursulines de Saint Charles venait moins d'une pédagogie spécifique que de l'intérêt porté à la personne. Les Filles du Sacré Cœur de Jésus, fondées dès 1831, s'occupaient elles aussi d'enfants venant des milieux aisés qui avaient bénéficié des réformes économiques et administratives de Lombardie pour vivre dans une laïcité hostile à la religion.

En Toscane, la Restauration revalorisa le rôle scolaire de la religion, mais aussi le contrôle de l'État sur l'éducation. Les Frères des Écoles chrétiennes (Scopoli) suppléèrent à la faiblesse de l'enseignement primaire public dans un strict respect de la loi. L'éducation des sourds-muets se développa à Sienne où le père Tommaso Pendolà préconisait le recours à la méthode labiale afin de faciliter leur insertion sociale et d'éviter dans leur esprit une matérialisation des idées. La Toscane vit naître la réflexion pédagogique du catholicisme libéral. Gino Capponi s’y opposait au rigorisme des Jésuites et Raffaele Lambruschini insistait sur les nouvelles obligations de la famille en matière d'éducation des enfants. Ils avaient tous les deux une haute conception du rôle de la femme dans la société qui les incitait à valoriser son rôle d'éducatrice. La pédagogie de ce courant " néo-guelfe » préconisait aussi une formation du caractère reposant sur l'autonomie de l'élève et sur son dynamisme intérieur; mais elle eut peu d'influence sur les nouvelles congrégations religieuses, à l'exception des initiatives scolaires des pères " scolopi ». La Toscane ne donna d'ailleurs naissance qu'à deux nouvelles congrégations religieuses féminines : les Augustiniennes Filles du Crucifix à Livourne en 1836 et les Sœurs "Stimmatine » à Florence en 1850.

La législation scolaire napoléonienne survécut en principe dans les duchés où les écoles de secours mutuel se répandirent à l'initiative des gouvernements de la Restauration. À Modène, François IV de Lorraine-Habsbourg s'adressa à la congrégation véronaise des Filles de Jésus qui ouvrit des écoles de charité dans 
les principales villes du duché. Leur implantation donna lieu à de vives polémiques entre le pouvoir civil qui entendait les maintenir sous son autorité et l'évêché qui prétendait les en soustraire. Après les insurrections de 1831, le duc souhaita enraciner le consensus à son régime par un contrôle strict de la société qu'il s'efforça d'exercer en multipliant les institutions charitables et enseignantes. Il fit appel aux Sœurs de la Charité de Jeanne-Antide Thouret qui s'étaient installées à Naples à l'époque de Murat. Ces dernières essaimèrent dans le duché puis dans les Légations pontificales où elles rejoignirent des fondations autonomes des Filles de Jésus. Les enseignements de Severino Fabriani au séminaire de Modène s'efforçaient de donner une rigueur scientifique à l'apologétique moderne pour mieux combattre la pensée des Lumières ; mais le prêtre était résolument engagé dans la vie sociale et il fut un des pionniers de l'éducation des sourds-muets en Italie. Il confia l'institut féminin de Modène aux Filles de la Providence et leur imposa la méthode de l'alphabet des signes de l'abbé de l'Épée ; mais il considérait la langue comme l'instrument indispensable à toute prise de conscience de l'ordre moral et spirituel de la religion. La pédagogie de Fabriani ignorait les orientations libérales du mouvement «néo-guelfe ». Elle s'inspirait plutôt d'une tradition salésienne où la communauté éducative s'identifiait à celle de la famille. Le maitre s'adressait à l'affectivité de l'enfant plus qu'à la formation rationnelle de son esprit.

Marie-Louise maintint à Parme un strict contrôle de l'État sur l'enseignement. Les anciennes congrégations religieuses enseignantes reprirent leurs activités, mais à condition d'ouvrir une école pour les filles pauvres à côté de leur pensionnat payant. Ce fut le cas des Ursulines, des Bénédictines et des Capucines dont les établissements de Parme, Plaisance et Guastalla furent subventionnés par l'État. Après les insurrections de 1831, l'enseignement public reçut une orientation résolument confessionnelle, mais sous un contrôle toujours strict des autorités ducales. Les Dames du Sacré-Cœur de Sophie Barat furent introduites à Parme en 1833 et les Filles du Sacré-Cœur de Jésus en provenance de Bergame dix ans plus tard. Par contre, l'enseignement public s'ouvrit largement aux nouvelles méthodes pédagogiques de Ferrante Aporti après 1848. La politique scolaire des duchés de Parme et de Modène fut donc caractérisée sous la Restauration par une cléricalisation progressive des enseignements dans des institutions religieuses qui échappaient à l'ordinaire diocésain et restaient étroitement soumises au pouvoir civil.

Les États pontificaux avaient été durement frappés par la confiscation des biens ecclésiastiques et par les prélèvements fiscaux de l'administration napoléonienne. L'une des principales préoccupations des papes fut de réorganiser une instruction publique démantelée par la suppression des ordres religieux en créant un système centralisé d'enseignement ; mais la réforme échoua devant l'opposition conservatrice des «zelanti » et l'instruction primaire fut confiée aux Frères des Écoles chrétiennes ainsi qu’à des instituts privés. Le gouvernement de Grégoire XVI fit appel à des congrégations féminines enseignantes, inaugurant une politique 
qui se poursuivra jusqu'en 1870. Les Sœurs de la Charité ouvrirent leurs écoles à Santa-Maria-in-Cosmedin, dans le quartier Ripa de Rome et elles furent suivies par les Sœurs de Saint-Joseph, dont le pensionnat payant comportait une annexe gratuite pour les plus démunies près de San Lorenzo dans le quartier du Monte. La curie romaine sollicita également la congrégation des Filles de la Divine Providence pour les enfants pauvres du quartier Regola et celle des Sœurs de l'Apostolat catholique qui recueillait les orphelines. Les Sœurs de Sainte Dorothée, congrégation génoise de tradition paulinienne, ouvrirent une école primaire payante et une autre gratuite pour les filles pauvres du quartier Saint-Ange. Pendant le pontificat de Pie IX, huit congrégations féminines enseignantes fondèrent de nouvelles écoles à Rome. Les Filles du Sacré-Cœur de Jésus s’installèrent au quartier du Monte en 1847 et les Sœurs de Saint Vincent de Paul multiplièrent les asiles au Trastevere, au Ponte et près de la Trinité-aux-Monts. An cours des années 1850 arrivèrent à Rome les Filles de la Croix de Saint André, une congrégation enseignante fondée en France dans la Vienne par Jeanne Bichier des Anges et André Fournet, qui s'installèrent dans le quartier du Campomarzo. Elles furent suivies par les Sœurs de Saint-Joseph de Cluny, dont les activités missionnaires n'avaient pas effacé la vocation d'enseignement, et qui créèrent des écoles au Monte. Les Sœurs de la Providence, fondées à Portieux dans les Vosges, ouvrirent leurs écoles pour les filles du peuple dans le quartier du Borgo, à l'intérieur de la cité léonine. En provenance de Belgique, les Frères de Notre-Dame de la Miséricorde s'adressèrent aux enfants pauvres et les Sœurs de la Providence et de l'Immaculée Conception se virent attribuer pour leurs enseignements des locaux situés au Trastevere où les Oblates augustiniennes de Saint Pascal étaient déjà installées depuis 1825. Ainsi, dix-sept institutions religieuses enseignantes arrivèrent à Rome entre 1815 et 1870. La seule congrégation née dans le Latium fut celle des Adoratrices du Sang du Christ, fondée en 1834 à Frosinone. La curie romaine entendait combattre la diffusion des doctrines philosophiques rationalistes dans la jeunesse et elle restitua l'enseignement secondaire aux ordres religieux traditionnels : Frères des Écoles Chrétiennes, Clercs réguliers somasques et surtout Jésuites. Les études du "Colleggio romano" étaient gratuites; mais elles connurent une crise qui se solda par la baisse du nombre d'élèves externes. Les Dames du Sacré-Cœur représentaient avec les Ursulines la principale congrégation féminine d'enseignement secondaire à Rome. Elles étaient arrivées de Turin en 1828 pour fonder une école payante destinée aux filles de l'aristocratie à la Trinité des Monts, avec une annexe gratuite au Trastevere.

Dans les Légations pontificales, le déclin des activités manufacturières textiles de Bologne avait provoqué la perte de nombreux emplois dans l'artisanat et accentué la paupérisation des classes populaires. Le problème de l'instruction des enfants pauvres retenait l'attention de l'archevêque Carlo Oppizoni qui souhaitait promouvoir un renouveau religieux. L'enseignement secondaire fut confié aux Barnabites qui remplacèrent les Jésuites. Les anciens couvents furent rouverts 
et l'ordre salésien de la Visitation Sainte-Marie reprit ses activités d'enseignement pour les filles de la noblesse dès 1818 . L'instruction primaire féminine était dispensée à Bologne par des institutrices laïques dans des écoles de la Providence étroitement contrôlées par l'archevêché. Elles furent confiées aux Sœurs de la Charité de Jeanne-Antide Thouret an cours des années 1840. L'ordre de la Visitation était de stricte clôture et il ne pouvait accueillir que quelques pensionnaires. L'arrivée à Bologne des Sœurs de Sainte Dorothée, fondées en 1855 par Paola Frassinetti, permit d'en recevoir plusieurs dizaines. À la frontière de l'Émilie, Elisabetta Renzi fondait en 1839 à Rimini les Institutrices pieuses de Notre-Dame des Sept Douleurs. Les problèmes éducatifs préoccupaient de larges secteurs du monde ecclésiastique des Légations dans des provinces où l'enseignement mutuel avait connu une certaine diffusion au début de la Restauration. Ces écoles à la pédagogie lancastérienne se répandirent dans les Marches et en Ombrie; mais elles furent vite suspectes aux autorités romaines qui les interdirent par la constitution Quod divina sapientia de Léon XII. Les évêques des Marches leur opposèrent une instruction primaire fortement imprégnée de morale chrétienne. En Ombrie, l'évêché de Spolète confia ses écoles aux Frères des Écoles Chrétiennes dès 1820. L'action conjuguée des écoles paroissiales et des congrégations religieuses enseignantes contribua à améliorer le taux d'alphabétisation qui atteignit $57 \%$ de la population de Rome en 1870 , soit près du double de la moyenne nationale italienne ; mais l'hostilité des autorités pontificales et épiscopales aux méthodes de l'enseignement mutuel et aux orientations pédagogiques libérales du mouvement néo-guelfe retarda la diffusion d'une nouvelle conception de l'enfance et freina l'insertion des couches populaires dans le tissu économique productif. Le retard technologique de l'artisanat et de l'agriculture uni au mauvais fonctionnement de l'administration restèrent les causes profondes de la stagnation économique des États du Saint-Siège.

L'insertion des Sœurs de la Charité de Jeanne-Antide Thouret dans le tissu social et religieux napolitain se heurta au poids de la tradition. La restauration des Bourbons les priva de l'appui des autorités civiles dont elles avaient bénéficié sous l'Empire. Les constitutions de la congrégation approuvées par Rome en 1819 rappelaient les règles fondamentales de la vie monastique. La Supérieure générale restait soumise à l'autorité diocésaine, tout en conservant son autonomie de gestion. Le meilleur atout de l'institution restait sa compétence en matière d'enseignement et d'assistance charitable. Ses écoles apprenaient à lire, à écrire, à compter et elles dispensaient une instruction d'économie domestique. Son pensionnat payant de Regina Cœli, réservé aux filles de la noblesse, innovait en préparant les élèves à leur futur rôle de maîtresse de maison. La formation spirituelle se distinguait d'un simple catéchisme par un effort de réflexion qui précédait l'administration des sacrements. Dans le royaume des Deux-Siciles, la situation fut plus favorable aux Jésuites que dans les États du nord de l'Italie marqués par l'influence joséphiste. Certes, ils ne purent retrouver leurs collèges de Sicile occupés par les frères des Écoles Chrétiennes; mais ils en ouvrirent de nouveaux 
à Palerme, Messine et Catane. L'alliance du trône et de l'autel se traduisit à Naples par une large cléricalisation du corps enseignant et par une préférence pour les modèles traditionnels d'inspiration jésuitique. Les nouvelles congrégations étaient rares et celle des sœurs de la Charité se tourna vers l'Italie centrale pour se développer. Les monastères restaient très majoritaires dans le royaume et on en dénombrait encore deux cent soixante-quinze en 1861, sans compter ceux de Sicile.

La spiritualité des nouvelles institutions religieuses s'inspirait des sources traditionnelles jésuites et salésiennes, auxquelles s'ajoutaient les héritages de Vincent de Paul, de Philippe Néri et d'Alphonse de Liguori. Ainsi, le Regole di vita d'Annunciata Cocchetti, fondatrice d'une des congrégations de Sainte Dorothée, se réclamait d'une rigueur ignatienne tempérée par la souriante humilité salésienne et par la piété accessible d'Alphonse de Liguori. Le noviciat de la congrégation des Filles du Sacré-Cœur de Jésus s’inspirait des «Exercices spirituels » ignatiens dans la discipline de la volonté, l'exercice permanent de la vertu, la pratique de l'examen de conscience et de la fréquente communion. L'influence des Jésuites était également perceptible dans les «Constitutions » du père Ludovico Pavoni pour les filles de Marie Immaculée et dans celles de Caterina Cittadini destinées aux ursulines de Somasca. La spiritualité ignatienne se mêlait encore à l'esprit franciscain dans les « Constitutions » des frères hospitaliers de l'Immaculée Conception de Luigi Monti où l'on percevait l'empreinte de la piété d'Alphonse de Liguori. Les congrégations enseignantes de Vérone s'inspiraient elles aussi d'une tradition ignatienne qui insistait sur la mission d'éducation de l'Église ; mais elles s'ouvraient aussi à la nouvelle culture catholique française d'un Lamennais ultramontain. La pensée salésienne jouait un rôle déterminant dans la spiritualité congréganiste et les règles édictées par Maddalena di Canossa aux Filles de la Charité s'inspiraient de l'expérience religieuse de la Visitation. La foi totale en Dieu impliquait un idéal de perfection vécu au quotidien dans l'humilité. La spiritualité salésienne était également présente dans les écrits de l'évêque Luigi Biraghi et de Teresa Eustochio Verzeri qui concevaient les voies de la sanctification comme une ascèse de la vie ordinaire. Elle imprégnait aussi un style d'éducation fondé sur un dialogue ouvert et affectueux, notamment dans la congrégation des sœurs de la Charité de Jeanne-Antide Thouret qui essaimait en Italie centrale à partir du royaume de Naples. Dans la tradition salésienne, la communauté éducative s'identifiait à celle de la famille. Le maître s'adressait à l'affectivité de l'enfant plus qu'il ne veillait à la formation d'un esprit rationnel. L'enseignement individualisé était attentif à la personnalité de l'élève et une règle inspirée des Frères maristes de Marcellin Champagnat voulait que la prévention précédât la répression. On retrouvait l'esprit de Vincent de Paul dans les "Constitutions » des Sœurs de Sainte Marcelline et la pensée d'Alphonse de Liguori alimentait une piété très éloignée de l'austère élitisme du jansénisme.

La politique de la Restauration misait sur un besoin d'expiation des impiétés révolutionnaires que la spiritualité des congrégations religieuses identifiait au rachat des péchés du monde et au sacrifice de la Croix. Elles compensaient leur 
absence d'initiatives spirituelles nouvelles par une forte sensibilité pratique qui se manifestait dans un apostolat d'assistance et d'éducation. La tradition charitable de Vincent de Paul était conçue comme une participation au mystère du Christ rédempteur et les malades représentaient les membres précieux de son corps souffrant. Cette spiritualité soulignait l'humanité du Christ et de la Passion. Elle se mêlait intimement à l'apologie des réparations dues à l'Église pour les blessures qui lui avaient été infligées. Elle se manifestait par un retour aux valeurs traditionnelles de la Contre-Réforme sous une forme critique à l'égard du protestantisme et du jansénisme. La théologie de la Croix de Rosmini s'exprimait dans l'exaltation et le sacrifice ; mais ce christocentrisme restait dénué des mollesses mystiques et des complaisances intimistes d'un romantisme christologique et l'amour du Christ permettait d'élever le service des autres au-dessus d'une simple action philanthropique. Les nouvelles congrégations rénovaient les valeurs et le contenu de la tradition où elles avaient mûri en se rattachant à la vision rosminienne de la charité. L'éducation en devenait une des formes les plus nobles lorsqu'elle réparait les dommages causés dans les âmes par le rationalisme des Lumières, si bien que la catéchèse restait étroitement unie à la formation civile. L'accent était mis sur une nouvelle évangélisation d'un monde qui devait être fondamentalement reconstruit. Le refus de la clôture confirmait cette vocation active liée au souci de la pastorale et les nouvelles congrégations reflétaient l'importance du rôle de la femme comme protagoniste de l'Église dans la reconquête de la société. Les religieuses « canossiennes » ne considéraient d'ailleurs pas l'enseignement comme leur activité principale et elles insistaient davantage sur un apostolat qui leur permettait de suivre leurs élèves après leurs études au moyen d'une assistance en cas de maladie et par des retraites spirituelles. Cette mission auprès des femmes impliquait un lien d'autant plus étroit avec la paroisse que la législation scolaire en Lombardie-Vénétie autrichienne faisait du curé le responsable des écoles. Les Sœurs de Sainte Dorothée confiaient même leurs élèves à des assistantes laïques qui les réunissaient périodiquement pour la catéchèse dans l'intention d'associer la femme à l'apostolat de l'Église.

L'indifférentisme des intellectuels, les distances prises avec l'Église par les classes bourgeoises et le déclin de la pratique religieuse dans les couches populaires étaient les symptômes les plus inquiétants de l'impiété. Il devenait nécessaire de ramener le Christ dans la société par l'éducation et la catéchèse de la jeunesse. Les écoles de charité des nouvelles congrégations enseignantes étaient gratuites pour les filles pauvres et ouvertes tous les jours sans obligation d'assiduité. Elles laissaient aussi aux élèves la possibilité d'aider leurs parents, ce qui était très apprécié des familles. L'organisation scolaire variait selon les institutions, les classes des écoles "canossiennes " comptant deux institutrices pour trente élèves et celles des Sœurs "Stimmatine " une seule pour soixante. L'âge des élèves allait généralement de cinq à douze ans. Les règlements demandaient d'apprendre à lire et à écrire ; mais ils accordaient une place importante aux travaux domestiques. 
Bien que persuadées de la nécessité de l'instruction pour les filles, les Sœurs de Sainte Dorothée jugeaient essentielles la pratique des vertus chrétiennes et l'habileté domestique afin de mieux les préparer à la vie matrimoniale à laquelle elles étaient destinées. La principale caractéristique des écoles "canossiennes » résidait dans un binôme "enseignement primaire-travail » qui traduisait un souci particulier pour la préparation à la vie professionnelle. Les congrégations " canossiennes » et celles des sœurs de Sainte Dorothée se préoccupaient de la formation d'institutrices rurales laïques ou ecclésiastiques. Elles devaient faire preuve d'affabilité, de douceur et de disponibilité dans leurs enseignements.

Les écoles de charité souhaitaient dépasser le stade de l'alphabétisation et offrir à leurs élèves un moyen de promotion sociale. L'accent était sans doute mis sur une formation religieuse capable de reconstituer le tissu social chrétien déchiré par les événements révolutionnaires, mais l'instruction comportait aussi une formation professionnelle, les oratoires n'étant plus adaptés à la situation nouvelle. Le travail artisanal était considéré comme un facteur d'épanouissement personnel et d'intégration dans la vie civile, même si les congrégations entendaient protéger les jeunes des dangers moraux encourus sur leur lieu de travail. L'instruction devenait ainsi un moyen d'intégration des classes populaires dans la société et cette adaptation à l'évolution sociale n'était pas étrangère au succès des nouvelles institutions religieuses. Elles restèrent pourtant étrangères au mouvement pédagogique de leur époque et le contenu de leur enseignement évolua peu : catéchisme, lecture, écriture, rudiments de calcul et travaux domestiques. Les bonnes manières cultivaient les vertus et s'efforçaient de combattre les erreurs propagées dans le peuple par le rationalisme. Les procédés didactiques restaient les mêmes, avec une prépondérance des abécédaires, des bouliers et des vies de saints. Les seules exceptions concernaient les frères des Écoles chrétiennes qui enseignaient aussi le dessin géométrique à des fins de formation professionnelle. Leur pédagogie influença l'enseignement des sœurs de Sainte Dorothée, de Saint Charles et de Saint Joseph qui privilégiait la règle du silence dans leurs écoles pour rendre les élèves plus attentives. Les manuels en usage ne se modernisèrent que très lentement avec l'adoption de ceux de Francesco Soave, un père somasque du XVIII ${ }^{\mathrm{e}}$ siècle chargé par Joseph II de la rénovation des écoles de Lombardie. Toutefois, le souci d'une dimension formatrice dépassant la seule assistance charitable, l'abandon des pratiques coercitives au profit du dialogue et l'adoption d'une éducation personnalisée s'inspirant du modèle familial représentaient d'incontestables innovations dans les nouvelles congrégations.

L'enseignement secondaire restait fortement influencé par le ratio studiorum jésuite réformé en 1832. La langue et la littérature nationales étaient devenues des disciplines principales du cycle des humanités, au même titre que le latin et le grec ; l'histoire, la géographie et l'arithmétique restaient des matières accessoires. Le cycle philosophique comportait désormais des mathématiques et des sciences 
naturelles, la référence aristotélicienne étant supprimée ; mais la réforme fut critiquée pour ses insuffisances, la philosophia perennis et les langues classiques conservant un rôle majeur dans le cursus. L'enseignement des Barnabites de Bologne était précédé d'un cycle d'études au gymnase, essentiellement consacré à l'apprentissage du latin. Cinq classes de collège venaient ensuite, les trois premières insistant sur la grammaire, les deux autres sur les humanités et la rhétorique. Les humanités classiques restaient donc très largement prépondérantes et la philosophie inexistante. Les collèges des clercs réguliers de Saint Paul enseignaient toutefois la langue et la littérature italienne, Dante, Pétrarque et le Tasse figurant au programme. L'enseignement des pensionnats des ursulines de Saint Charles se limitait à la calligraphie, à l'arithmétique, à la grammaire, à la couture et au catéchisme. Celui des sœurs de Sainte Dorothée y ajoutait la langue française, l'histoire, la géographie ainsi que des notions d'histoire naturelle, de géométrie et de physique. L'objectif était de dépasser la conception des couvents qui faisait de l'enseignement un simple ornement, afin de former des mères de famille mieux armées pour affronter les exigences sociales de leur temps. Le programme des collèges des Sœurs de Sainte Marcelline était à peu près identique, avec un enseignement rudimentaire d'allemand, de chant et de dessin. L'enseignement était un moyen de promotion de la femme, mais la pratique des œuvres et des vertus chrétiennes restait la meilleure voie pour y parvenir. Par contre, les études secondaires proposées par les Dames du Sacré-Cœur s'inspiraient largement du ratio studiorum jésuite. Le collège romain de la congrégation de Sophie Barat affichait de grandes ambitions. Son "Plan d'éducation " de 1826 se contentait d'une pédagogie communautaire et dénonçait les dangers d'une éducation individuelle ; mais le règlement de 1852 allait beaucoup plus loin en regroupant les élèves par classes et en organisant la vie scolaire en années d'enseignement séparées par des vacances. Le cursus comportait un «Cours préliminaire » de deux ans qui dispensait un enseignement primaire et une première formation chrétienne aux fillettes âgées de cinq à sept ans. Le "Cours régulier » composé de cinq classes était accessible aux élèves sachant lire et écrire. Le programme prévoyait l'instruction religieuse, la grammaire, la littérature italienne et française, la calligraphie, la rhétorique et l'arithmétique, un enseignement d'histoire sainte et profane avec chronologie et mythologie, la géographie, le dessin, la peinture, la musique, l'économie domestique et des travaux manuels. La «Classe supérieure » enfin, perfectionnait en deux ans les connaissances précédemment acquises et innovait profondément avec un cours de logique, de métaphysique, d'éthique et de morale. L'objectif de cet enseignement était de permettre aux jeunes filles de l'aristocratie de s'exprimer parfaitement en italien comme en français et de posséder une bonne connaissance de la littérature nationale pour agrémenter leur conversation. L'importance prêtée à l'histoire et à la géographie incitait à des rapprochements entre les époques et à un élargissement de l'horizon de la pensée. L'enseignement de la philosophie était une nouveauté destinée à améliorer le raisonnement et le jugement pour mieux combattre l'indifférentisme religieux. Le succès des Dames 
du Sacré-Cœur permit à leur institut romain de la Trinité-aux-Monts de doubler le nombre de ses élèves entre 1840 et 1870 .

La bibliothèque des nouvelles congrégations religieuses reflétait leur volonté de former des mères vertueuses capables de remplir leur mission d'éducation au sein de la famille. Nombre de ces ouvrages portaient toujours les traces de la misogynie diffuse de Fénelon et de Rousseau, et ils la propageaient sous une forme plus accessible à leurs lectrices : dialogues édifiants, correspondances intimes, conseils d'une mère à sa fille, éloge de l'amour conjugal, apologie des vertus des femmes célèbres. Le traité De l'éducation des femmes de madame Campan, qui affirmait la nécessité d'instruire les filles en musique, dessin, histoire et géographie, donna naissance à plusieurs versions italiennes. Les unes s'efforçaient d'en vulgariser le texte, d'autres lui adjoignaient des conseils de morale à la manière des Conversations d'Émilie de madame d'Epinay. Les œuvres de madame de Genlis furent largement diffusées dans la péninsule et leur succès venait de contes moraux plusieurs fois réédités à Milan et à Naples, villes sous domination autrichienne et bourbonienne. Le récit évoquait les risques encourus par un amour détourné de ses projets initiaux dans une famille où le mari était présenté comme l'instituteur de son épouse. Le roman Adèle et Théodore, qui invitait, dès 1782, les dames de l'Ancien Régime à renouer avec leurs anciennes vertus domestiques, était présenté aux lectrices italiennes sous forme de lettres sur l'éducation dans un recueil d'œuvres utiles pour la jeunesse. Le succès de Jean-Nicolas Bouilly en Italie ne devait rien à son passé de révolutionnaire, ami de Barnave et de Mirabeau. Il était lié à son œuvre dramatique Léonore (1798) qui devait inspirer le livret de l'opéra Fidelio de Beethoven. Cet éloge de l'amour conjugal, destiné à des jeunes filles qui n'avaient guère le choix qu'entre la vocation religieuse et le mariage, donna lieu à de multiples publications dans la péninsule sous forme de conseils d'une mère à sa fille et de récits moraux destinés aux jeunes épouses. Le Magasin des enfants de madame Leprince de Beaumont connut une version italienne plusieurs fois rééditée à Venise. Ce roman pour la jeunesse et son fameux conte La Belle et la bête, modèle de rédemption par l'amour, parut à Milan dans la version simplifiée d'un dialogue entre une éducatrice et ses élèves. De même, le traité d'Aimé Martin De l'éducation des mères de famille qui attribuait à la femme un rôle majeur dans l'évolution de la civilisation du genre humain, eut beaucoup de succès dans la péninsule. Par contre, les romans de la duchesse d'Abrantès ne survécurent guère à leur auteure et les lectrices italiennes durent se contenter des vies de femmes célèbres d'Adélaïde Dufresnoy, en ignorant les ouvrages d'éducation de cette auteure. Il est vrai qu'après 1815 , le salon de madame Dufresnoy était devenu l'un des cénacles de l'opposition libérale à Paris.

Après Fénelon, Rousseau fut l'écrivain de l'époque prérévolutionnaire qui exerça le plus d'influence sur la littérature d'éducation féminine au XIX ${ }^{\mathrm{e}}$ siècle. Il soulignait la diversité biologique de la femme dans son Discours sur l'origine et les fondements de l'inégalité des hommes et proclamait son infériorité intellectuelle dans l'Émile. On sait que Joseph De Maistre lui emprunta beaucoup, tout 
en renversant ses démonstrations sur le mythe du " bon sauvage "; mais il partageait ses conceptions au sujet de la femme, le courant contre-révolutionnaire qu'il incarnait souhaitant restaurer une hiérarchie traditionnelle dans la famille. Cette volonté de prendre le contre-pied des Lumières et de la Révolution française était manifeste dans l'Éloge historique de madame Élisabeth de France, un ouvrage d'Antoine Ferrand largement diffusé dans l'Italie de la Restauration. L'auteur était un conseiller au Parlement de Paris qui avait émigré en 1789 avant de devenir pair de France sous Louis XVIII. Il citait son héroïne en exemple pour ses vertus chrétiennes qui pouvaient pousser le dévouement à Dieu et au roi jusqu'au sacrifice.

L'un des ouvrages les plus répandus dans les milieux catholiques italiens était le Trattato dell'educazione dei fanciulli de Cesar de La Luzerne, publié à Rome en 1852. L'auteur était un évêque français d'Ancien Régime qui avait contribué à la rédaction de l'édit royal donnant un état civil légal aux non-catholiques du royaume de France. Rentré d'émigration en 1814, pair de France et ministre d'État, il avait fait preuve de tolérance sous la Restauration en reconnaissant la capacité civile aux établissements de tous les cultes ; mais la plupart des références théologiques de la littérature destinée aux femmes étaient strictement conservatrices. Les livres de Jean Joseph Gaume, traduits en italien dans les années 1850, concernaient la préparation au sacrement de la communion ; mais l'auteur était connu pour son pamphlet Le Ver rongeur des sociétés modernes, ou le Paganisme dans l'éducation qui avait suscité de vives polémiques. Une intransigeance doctrinale comparable animait Il mentor dei fanciulli et le Vita di alcune giovinette, publiés en 1830 et 1831 à Venise et Milan sous domination autrichienne. Leurs auteurs étaient deux prêtres réfractaires français, l'abbé Blanchard et le père Guy Toussaint Caron. Le premier passait pour le théoricien de la résistance au concordat napoléonien de 1801 et il était l'un des fondateurs du schisme de la Petite Église ; le second avait multiplié les œuvres de charité en Angleterre puis en France, et on lui devait une Histoire des confesseurs de la foi dans l'Église gallicane à la fin $d u \mathrm{XVIII}^{e}$ siècle, véritable mine d'informations sur l'émigration. L'ouvrage italien le plus diffusé dans les familles catholiques de la péninsule restait pourtant les Regole di vita per una giovinetta de l'évêque Vincenzo Maria Strambi, plusieurs fois réédité entre 1821 et 1871 .

Les livres de lecture des écoles congréganistes enseignantes féminines étaient rédigés par des ecclésiastiques et n'avaient pas que des finalités éducatives. $\mathrm{Au}$ premier rang des auteurs figuraient l'abbé Louis Bautain et le père théatin Gioacchino Ventura. Impressionné par la critique kantienne, Bautain affichait son hostilité au rationalisme et proclamait la capacité de la foi à parvenir seule aux vérités fondamentales; mais il s'inclina devant la condamnation romaine du fidéisme (1838), l'Église enseignant que la raison pouvait atteindre la vérité dans l'ordre des principes naturels. Ventura devint le chef de file de la philosophie catholique en Italie avec son De jure ecclesiastico (1826); mais sa célébration de l'alliance de la religion et de la liberté, puis son engagement dans les mouvements révolutionnaires de 1848 le contraignirent à s'exiler à Paris où ses prédications 
lui valurent une grande notoriété (La Raison philosophique et la raison catholique). Les ouvrages de Bautain furent largement diffusés en Italie dans des adaptations dues au père Francesco Pricolo. Ils concernaient les problèmes posés par l'âge critique à toute adolescence chrétienne, donnaient des conseils aux novices et aux jeunes filles désireuses de se marier contre la volonté de leurs parents. Ventura était plein de prévention contre la culture féminine, tout en reconnaissant à la femme une supériorité morale qui l'autorisait à jouer un rôle primordial dans la famille. Les écrits du théologien génois Giuseppe Frassinetti, pleins de dévotion pour le pape et pour l'Église, voulaient aider les adolescents et les jeunes mariés à vivre leur foi, au même titre que les pensées du père Tommaso Pendolà, publiées dans un recueil par Gian Battista Fenoglio. Le prêtre turinois Pietro Tarino proposait enfin la Vierge Marie comme modèle de femme chrétienne. L'idéal catholique d'une épouse exemplaire, responsable de l'éducation morale et religieuse de ses enfants, s'exprimait dans une littérature édifiante mais imbue de la nécessité d'une éducation féminine capable d'éveiller conscience et discernement. Sans doute faisait-elle partie d'une tactique silencieuse d'enveloppement qui dissuadait les jeunes filles d'échapper à la vie conjugale; mais cette condition qui nous apparait aujourd'hui comme une forme d'esclavage supérieur n'était pas vécue dans la passivité et la résignation. Le XIX siècle vit en effet s'imposer le rôle de la mère dans l'éducation des enfants et beaucoup le ressentirent comme une promotion. Les lectures de romans moralisateurs et de livres de dévotion accentuèrent par contre la tendance italienne à lui attribuer des vertus qui transcendaient progressivement ses devoirs maternels pour investir la société tout entière. Le mythe de la mamma s'imposait.

La Restauration fut dominée en Italie par une tendance contre-révolutionnaire et par une reconquête religieuse; mais elle ne s'identifia pas à une restauration de l'Ancien Régime. L'Église retrouva des prérogatives étendues en matière d'éducation et d'assistance charitable qu'elle exerça sous un contrôle strict de l'État. La pensée pédagogique chrétienne se préoccupait d'éducation populaire et du rôle de la femme dans la société, ses tendances libérales se distinguant du conformisme et du traditionalisme par une place plus importante accordée à la personnalité de l'enfant. L'analphabétisme régressait lentement et l'école du peuple progressait. Les nouvelles congrégations religieuses jouèrent un rôle important dans ces mouvements qui traduisaient une évolution du monde catholique italien. L'image absolutiste s'estompait au profit d'une conception humaniste du pouvoir plus compatible avec l'idéal national. La formation dispensée par les nouvelles institutions enseignantes n'en restait pas moins empreinte d'une atmosphère et de caractères propres à la vie religieuse. L'assistance charitable s'avérait inséparable d'une reconquête chrétienne qui s'insérait elle-même dans un combat idéologique contre le rationalisme. Les éducateurs italiens de sourds-muets opposaient ainsi au concept rationaliste de la méthode des signes un processus qui partait d'un constat réaliste pour s'élever vers l'objet mystique de la création divine. L'éducation congréganiste mettait l'accent sur la sensibilité aux valeurs morales et religieuses, sur le sérieux dans la vie, sur la préparation professionnelle et elle acceptait 
même une certaine ouverture sur le monde extérieur ; mais elle ne parvenait pas à s'affranchir d'une emphase excessive des vertus passives et d'un contrôle rigide des formes d'expression individuelle. Ces faiblesses restaient pourtant du domaine des moyens employés et l'objectif social dépassait largement la simple réaction cléricale décrite par bien des manuels d'histoire.

\section{Michel OSTENC \\ Professeur d'Université honoraire nmod@noos.fr}

\section{Bibliographie}

Arosio P., Sani R., Sulle orme di Vincenzo de'Paoli. Jeanne-Antide Thouret e le Suore della Carità dalla Francia rivoluzionaria alla Napoli della Restaurazione, Milan, 2001.

Ascenzi A., Il Plutarco delle donne. Repertorio della pubblicistica educativa e scolastica $e$ della letteratura amena destinate al mondo femminile nell'Italia dell'Ottocento, Macerata, Edizioni Università, 2009.

Ascenzi A., FAtTori G., L'alfabeto e il catechismo. La diffusione delle scuole di mutuo insegnamento nello Stato Ponfificio (1819-1830), Pise-Rome, 2006.

Chiosso G., Carità educatrice e istruzione in Piemonte. Aristocratici, filantropi e preti di fronte all'educazione del popolo nel primo '800, Turin, Società Editrice Internazionale, 2007.

Covato C., Venzo M. I. (éd.), Scuola e itinerari formativi dallo Stato Pontificio a Roma capital. L'istruzione primaria, Milan, 2007.

GAUDIO A., «La Famiglia e la scuola » e la tradizione moderata fiorentina, in Chiosso G., Scuola e stampa nell'Italia liberal. Giornali e riviste per l'educazione dall'Unità a fine secolo, Brescia, 1993.

GHizzoni C., Polenghi S. (éd.), L'altra metà della scuola. Educazione e lavoro delle donne tra Otto e Novecento, Turin, Società Editrice Internazionale, 2008.

Morandini M. C., Scuola e nazione. Maestri e istruzione popolare nella costituzione dello Stato unitario (1848-1861), Milan, Vita e Pensiero, 2003.

Pazzaglia L. (éd.), Chiesa e prospettive educative in Italia tra Restaurazione e unificazione, Brescia, 1994.

Petrocchi M., Storia della spiritualità italiana, Turin, 1996.

SANI R., "Spiritualità e ideali di vita religiosa femminile in Francia e in Italia tra Rivoluzione e Restaurazione ", Annali della Facoltà di Scienze della Formazione, (Macerata), 1, 2004, p. 337-354.

-, « Ad Maiorem Dei Gloriam ». Istituti religiosi, educazione e scuola nell'Italia moderna e contemporanea, Edizioni Università di Macerata, 2009.

SANI R. (éd.), Chiesa, educazione e società nella Lombardia del primo Ottocento. Gli Istituti religiosi tra impegno educativo e nuove forme di apostolato (1815-1860), Milan, 1996.

SANi R., Ascenzi A. (éd.), Vita religiosa, carità ed educazione nell'Italia dell'Ottocento. Rosalie Thouret e la fondazione della Provincia modenese delle Suore della Carità (1834-1853), Macerata, Alfabetica, 2007.

Veneruso D., Carisma e progettualità di Luigi Maria Monti e dell'Istituto dei Figli dell'Immacolata Concezione, Soveria Mannelli (Catanzaro), Rubbettino, 2009. 